\title{
Steady-State and Switch-Off Behavior of Upconversion in Er-Doped Fibers
}

\author{
D. KHOPTYAR AND B. JASKORZYNSKA* \\ Laboratory of Optics, Photonics and Quantum Electronics, Department \\ of Microelectronics and Information Technology, Royal Institute of Technology \\ Electrum 229, 16440 Kista, Sweden

\begin{abstract}
By measuring the metastable and the upconverted fluorescence in Er-doped fibers we determine rate of homogeneous upconversion as a function of the population inversion. The results confirm the prediction of our statistical model that at the same population inversion the upconversion rates under the steady-state and the switch-off conditions are different. The larger rate of the steady state upconversion is attributed to the pump enhanced redistribution of the excitation energy.
\end{abstract}

PACS numbers: 71.20.Eh, 78.20.Bh, 78.55.-m, 42.65.-k

\section{Introduction}

Concentration dependent upconversion caused by interactions between two excited Er ions is a gain limiting factor in erbium doped waveguide amplifiers and lasers. A proper description of this effect is especially important for design of short Er-doped devices that require high doping concentrations. It has been theoretically predicted [1], and we experimentally observed $[2,3]$ that for randomly distributed Er ions the rate of upconversion is not a linear function of the population inversion as it was assumed in the earlier used models describing Er-doped glass devices [4].

It was also predicted [1] and experimentally confirmed [2] that the steady-state upconversion rate depends not only on the population inversion but also on the combination of pump and signal powers were applied for reaching a given population level.

Those features are also well explained by our statistical model [5] that does not require Monte Carlo simulations as that of Ref. [1] but for the steady-state case provides a transcendental equation for the population inversion and the upconversion rate.

*corresponding author; e-mail: bj@imit.kth.se 
Recently, we have extended the model [6] to account for the switch-off decay after continuous wave $(\mathrm{CW})$ pumping. We found that at the same population inversions the switch-off upconversion is weaker than that of the steady state.

Here we present an experimental confirmation of the predicted effect. We also show that our model allows a fairly good, consistent fit to the experimental results if one disregards the influence of the excess clustering of Er ions that is not accounted by the model.

\section{Experimental method}

We have simultaneously measured powers of the transverse to the Er-fiber fluorescence from the metastable $(1550 \mathrm{~nm})$ and the second excited level $(980 \mathrm{~nm})$ using a pump at $1.48 \mu \mathrm{m}$.

The absolute values of the upconversion rates in the steady state and the switch-off regimes were determined using the methods that we proposed and described in $[7,8]$. The method for the switch-off upconversion rate is based on fitting characteristics obtained from $1550 \mathrm{~nm}$ and $980 \mathrm{~nm}$ fluorescence decays [7].

The shape of the steady-state upconversion rate as a function of population inversion was found as a ratio of $1550 \mathrm{~nm}$ and $980 \mathrm{~nm}$ fluorescence powers at different power levels of a CW pump. For the calibration of the steady-state curve the decay measurements were made for several levels of the pump power then the steady-state curve was fitted to the switch-off values at the beginning of the decay [8].

\section{Results and discussion}

The experimentally determined (dashed lines and circles) and the calculated (solid lines) upconversion rates as a function of the population inversion are shown in Fig. 1. The dashed lines are obtained from the decay measurements and the circles from the steady-state measurements. The two solid lines are calculated using our model $[5,6]$. The lower one corresponds to the switch-off case and shows how the upconversion rate decreases with the decaying population inversion of the metastable level. The upper curve shows how the upconversion rate depends on the population inversion under CW pumping. The upconversion rate is normalized with a lifetime of the metastable level, and the population inversion with its maximum value ( $c a .0 .7$ for $1.48 \mu \mathrm{m}$ pump).

One can see from the experimental results that accordingly to the model predictions the steady-state upconversion is stronger than that for the switch-off case. This effect can be explained as follows. In an amorphous glass matrix Er ions are distributed in an irregular way. In particular, part of them form clusters. The upconversion strongly depends on the distance between the excited ions, and in clusters it occurs with the orders of magnitude higher rate than for unclustered 


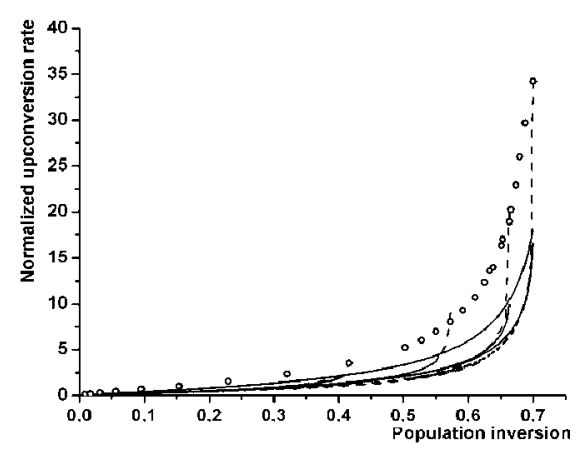

Fig. 1. The experimentally obtained (dashed lines and circles) and the calculated (solid lines) upconversion rates vs. population inversion of the metastable level. The upconversion rate is normalized with the metastable decay time, and the population inversion with its maximum value. The circles are experimental values of the steady-state upconversion rate for varying level of the $\mathrm{CW}$ pump at $1.48 \mu \mathrm{m}$. The upper solid line is the corresponding calculated dependence. The dashed lines are the experimental decays of the switch-off upconversion rate for four different levels of the initial population. The corresponding (lower) solid lines are the fitted theoretical decays.

ions. After the upconversion process all but one ions in a cluster relaxed to the ground state giving their excitation energy to the second excited level. This leads to hole burning in the excitation distribution where the ions are densest spaced. In the steady-state case both pumping and migration tend to refill the holes thus enhancing the overall upconversion rate. For the switch-off case only the migration effect is left and hence the upconversion is weaker. It should be pointed out that this difference cannot be described by macroscopic models because they do not take into account nonuniform excitation distribution on Er ions. The effect is also consistent with the earlier made [1,2] observation that the upconversion depends not only on the population inversion but also on how this inversion is realized.

In our model we assumed a random, volume uniform, ion distribution. The calculated steady-state upconversion rate (the upper solid line) is significantly lower than its experimental values. We attribute this to the fact that in the investigated fibers the percentage of clusters is much higher than that characteristic of the random distribution. This also strongly modifies the decay curves. The excess of clustering makes the beginning of the decay much steeper than predicted by our model.

This is because directly pumped clusters are the fastest channels of energy upconversion to the second excited level and therefore are a dominant source of $980 \mathrm{~nm}$ fluorescence. However, after switching-off the pump the contribution from the directly pumped clusters decays fastest as well (during several microseconds), while the upconversion between unclustered ions and migration re-excited clusters still keep populating the $980 \mathrm{~nm}$ level with initial rates (at high population in- 
versions) typically of the order of a millisecond or a sub-millisecond. The further decay is determined by the decay of the population inversion.

In order to eliminate this first-order contribution from the excess clustering we disregarded the initial $15 \mu$ s of the decay and under this approximation we were able to make a consistent fit to all the decay curves. The upconversion and migration radii, as well as the population inversion within uncertainty of the measurement, were used as the fitting parameters.

\section{Conclusion}

We have experimentally confirmed the prediction of our statistical model that for nonuniformly distributed Er ions the upconversion at the $\mathrm{CW}$ pumping is larger than that after the pump switch-off for the same population inversions. We attribute this difference to pump enhanced refilling of the spatial holes in the excitation energy distribution, which strongly increases the overall upconversion via re-excitation of the clustered ions.

Although the investigated fibers have much larger portion of clusters than assumed in our model we showed that the model allows to consistently fit the experimental results when the initial decays, strongest influenced by the excess of clustering, are disregarded.

\section{Acknowledgments}

The Swedish Foundation for Strategic Research (SSF) is gratefully acknowledged for supporting this research. The first author would like to thank Marcin Swillo for valuable discussions on the experimental techniques.

\section{References}

[1] J.L. Philipsen, A. Bjarklev, IEEE J. Quant. Electron. 33, 845 (1997).

[2] D. Bremberg, S. Helmfrid, B. Jaskorzynska, M. Swillo, J.L. Philipsen, B. Pálsdóttir, Electron. Lett. 14, 1189 (1999).

[3] J. Philipsen, J. Broeng, A. Bjarklev, S. Helmfrid, D. Bremberg, B. Jaskorzynska, B. Palsdottir, IEEE J. Quant. Electron. 35, 1741 (1999).

[4] P.C. Becker, Erbium-Doped Fiber Amplifiers: Fundamental and Technology, Academic Press, New York 1997, p. 191.

[5] S.V. Sergeyev, B. Jaskorzynska, Phys. Rev. B 62, 15628 (2000).

[6] S.V. Sergeyev, D. Khoptyar, B. Jaskorzynska, Phys. Rev. B 65, 233104 (2002).

[7] M. Swillo, D. Bremberg, B. Jaskorzynska, S. Helmfrid, J.L. Philipsen, in: Integrated Photonic Research, OSA Technical Digest, Optical Society of America, Washington DC 1999, p. 73.

[8] D. Khoptyar, B. Jaskorzynska, in: Proc. ECOC 2002, Vol. 1, Ed. P. Danielsen, Technical University of Denmark, Lyngby 2002, paper 2.2.6. 